Original Article

\title{
PREVENTION OF PEPTIC ULCERS BY CURCUMIN IN CHEMICALLY INDUCED OSTEOARTHRITIS
}

\author{
NASREEN SULTHANA ${ }^{*}$, VIJAYA K. ${ }^{2}$ \\ ${ }^{1}$ Faculty of Pharmacy, Pacific Academy of Higher Education and Research, Udaipur, Rajasthan, ${ }^{2}$ Professor and Principal, TKR College of \\ Pharmacy, Meerpet, Hyderabad, Telangana \\ Email: nasrpharma31@gmail.com \\ Received: 06 Feb 2017 Revised and Accepted: 24 Feb 2018
}

\begin{abstract}
Objective: The present study was carried out to investigate the role of curcumin in osteoarthritis when it is used as an adjuvant to diclofenac sodium.

Methods: Osteoarthritis (OA) was induced by administering nalidixic acid $400 \mathrm{mg} / \mathrm{kg}$. Animals (Female rats) were divided into 5 groups each containing 6 animals. Group I was considered as control, group II in which the animals were induced with osteoarthritis with nalidixic acid and were given no treatment. Group III in which osteoarthritis induced animals were treated with diclofenac sodium by the oral route. Group IV osteoarthritis induced animals were treated with the combination of diclofenac sodium and curcumin and group V animals were pre-treated with curcumin and then induced with osteoarthritis. Parameters like ulcer area, ulcer index, free acidity, total acidity, the volume of gastric juice were estimated. Histopathological studies were also carried out.
\end{abstract}

Results: The data of our study shows that nalidixic acid has not shown much effect on the gastric parameters in group II animals. The ulcer index, free acidity, total acidity and gastric juice volume were increased significantly $(\mathrm{p}<0.001)$ in group III and decreased in group IV animals ( $<<0.05$ ) when compared to group I control animals. Group V animals pretreated with curcumin have shown fewer incidences of gastric ulcers and other ulcerative parameters non significantly. Histopathology also suggests a low incidence of ulcers in group IV and group V.

Conclusion: It demonstrates that curcumin when used along with the conventional NSAIDs as an adjuvant therapy, has a role in treating osteoarthritis effectively.

Keywords: Osteoarthritis, Gastric ulceration, Nonsteroidal anti-inflammatory drugs, Curcumin

(C) 2018 The Authors. Published by Innovare Academic Sciences Pvt Ltd. This is an open access article under the CC BY license (http://creativecommons.org/licenses/by/4.0/) DOI: http://dx.doi.org/10.22159/ijpps.2018v10i4.24327

\section{INTRODUCTION}

Osteoarthritis (OA) is a pro-inflammatory degenerative disorder affecting millions of individuals in whole over the world [1]. It is the most common type of arthritis. Soreness and stiffness in joints, Heberden nodes [2] and pain are the common symptoms experienced in Osteoarthritis. Alleviating pain is the treatment goal in osteoarthritis treatment. Nonsteroidal anti-inflammatory drugs (NSAIDs) like diclofenac sodium, naproxen, indomethacin, and ibuprofen are conventionally used to reduce the pain.

Although these drugs help in reducing the pain they cause many adverse effects of which gastric ulceration is important which is one of the reasons for the increased mortality and morbidity. Curcumin is an active constituent available in the rhizome of Curcuma longa (Turmeric) which belongs to the family Zingiberaceae. Curcumin is thought to possess anti-inflammatory, antioxidant, anti-fungal, antiviral properties. It also suppresses the tumor formation and blood vessel formation.

Quinolone antibiotics are the drugs which act by inhibiting DNA gyrase and topoisomerase enzyme in bacteria. In addition to this activity, it degenerates articular cartilage due to their affinity towards the cartilage and bone. Blisters like lesions are formed on the cartilage followed by its degeneration after $24 \mathrm{~h}$ of its administration. This is evidenced by the appearance of nodules [3].

The present study was aimed to investigate the effect of curcumin when used with the standard drug in the treatment of Osteoarthritis and also its role in the prevention of gastric ulceration when animals are pretreated with it. Parameters like ulcer area, ulcer index, free acidity, total acidity, the volume of gastric juice were studied.
Histopathological study of the stomach was also carried out to determine the effect of curcumin.

\section{MATERIALS AND METHODS}

Animals

24 female Wistar rats weighing around 180-250 g were used for this study. The animals were accommodated in standard conditions of ventilation and temperature $\left(25 \pm 2^{\circ} \mathrm{C}\right)$, humidity $(60-70 \%)$ and light/dark condition (12/12). They were housed in pathogen-free conditions.

The animal procedures were conducted according to CPCSEA guidelines. Approval for animal studies was obtained from an animal ethical committee of TRR College of pharmacy with ethical clearance number: $2 /$ IAEC/TRRCP/2016.

\section{Chemicals}

Curcumin, nalidixic acid, dimethyl Sulfoxide were purchased from Hychem Laboratories, Hyderabad.

Nalidixic acid was administered at a single dose of $400 \mathrm{mg} / \mathrm{Kg}$ subcutaneously.

Diclofenac sodium was administered orally at a dose of $13 \mathrm{mg} / \mathrm{Kg}$ for 21 days. Curcumin was given orally at a dose of $200 \mathrm{mg} / \mathrm{Kg}$ for $21 \mathrm{~d}$.

\section{Experimental design}

The animals were allowed for stabilization or acclimatization for one week. Then they were grouped below 5 groups each containing 6 animals. 
Table 1: Experimental design and treatment of animals

\begin{tabular}{lll}
\hline S. No. & Group & Treatment \\
\hline 1 & I & Vehicle-treated \\
2 & II & Nalidixic acid \\
3 & III & Nalidixic acid+diclofenac sodium \\
4 & IV & Nalidixic acid+diclofenac sodium+curcumin \\
5 & V & Pretreatment with curcumin+nalidixic acid \\
\hline
\end{tabular}

Female rats $(n=30)$ were divided into five groups. Group I was considered as control. Group II, III and IV animals were induced with osteoarthritis with the administration of nalidixic acid (400 mg/kg) s. c. Group II was disease control group. Group III animals were treated with diclofenac sodium $(13 \mathrm{mg} / \mathrm{kg})$ p. o and group IV animals were treated with the combination of diclofenac sodium (13 $\mathrm{mg} / \mathrm{kg}$ ) p. o and curcumin (200 mg/kg) p. o. Group V animals were pretreated with curcumin $(200 \mathrm{mg} / \mathrm{kg})$ and then were administered with nalidixic acid (400 mg/kg) s. c.

Group I: Control group-Animals treated with vehicle

Group II: Disease control-Osteoarthritis induced animals with nalidixic acid

Group III: Standard group-Osteoarthritis induced animals treated with diclofenac sodium

Group IV: Test group-Osteoarthritis induced animals treated with diclofenac sodium and curcumin.

Group V: Pretreated group-Animals were pretreated with curcumin before the induction of Osteoarthritis.

\section{Estimation of ulcer index}

After the completion of collection of samples, the animals were sacrificed and their intact stomachs were isolated. The stomachs were opened through greater curvature and washed slowly under tap water to score the ulcer. The score of the ulcers formed was given as below:

0 = normal, $0.5=$ Red coloration, $1.0=$ Spot ulcers, $1.5=$ Hemorrhagic streaks, $2.0=$ ulcers $\geq 3 \leq 5,3.0=$ more than 5 ulcers [4]. Mean score of the ulcers was expressed as ulcer index. The number of ulcers per stomach was recorded and was compared with the control.

\section{Estimation of volume of gastric juice}

After isolating the stomach, the gastric juice was collected and gastric secretion studies were performed. The gastric juice was measured using measuring cylinder. After cleaning, the stomachs isolated were preserved in $0.1 \mathrm{M}$ phosphate saline buffer $(1: 4(\mathrm{w} / \mathrm{v})$, $\mathrm{pH}$ 7.4) prior to macroscopic examination and homogenization [5].

\section{Estimation of free acidity and total acidity}

After collecting the gastric juice, the collected juice was centrifuged at $1000 \mathrm{rpm}$ for $10 \mathrm{~min}$. The supernatant formed was separated and $1 \mathrm{ml}$ of it was collected and was made to $10 \mathrm{ml}$ by adding $9 \mathrm{ml}$ of distilled water. The resultant solution was titrated with $0.1 \mathrm{~N} \mathrm{NaOH}$. Topfer's reagent has used an indicator and was added till canary yellow color was observed. The amount or volume of $\mathrm{NaOH}$ required to get the color was noted and was considered as the value of free acidity. 2-3 drops of phenolphthalein were used as an indicator and were added to $\mathrm{NaOH}$ until the pink color was restored. This value corresponds to total acidity [5]. The values obtained were compared among test, control and standard groups.

Acidity $=$ Volume of $\mathrm{NaOH} \times$ Normality of $\mathrm{NaOH} \times 100 \mathrm{mEq} / \mathrm{l}$

\section{Statistical analysis}

The data were expressed as mean \pm S. D values. Results were analyzed statistically by One-way ANOVA followed by Dunnett's Test using standard statistical software package of Graph pad prism.7. The difference was considered significant if $* / \# \mathrm{p}<0.05, * * / \# \#$ $\mathrm{p}<0.001, * * * / \# \# \# \mathrm{p}<0.0001, * * * * / \# \# \# \# \mathrm{p}<0.00001$.

*All the groups were compared to the control

\#All the groups were compared to the disease control or the group with animals induced with osteoarthritis and are given no treatment.

\section{RESULTS}

Effect of various treatments on the ulcerative parameters in osteoarthritis (OA) induced rats:

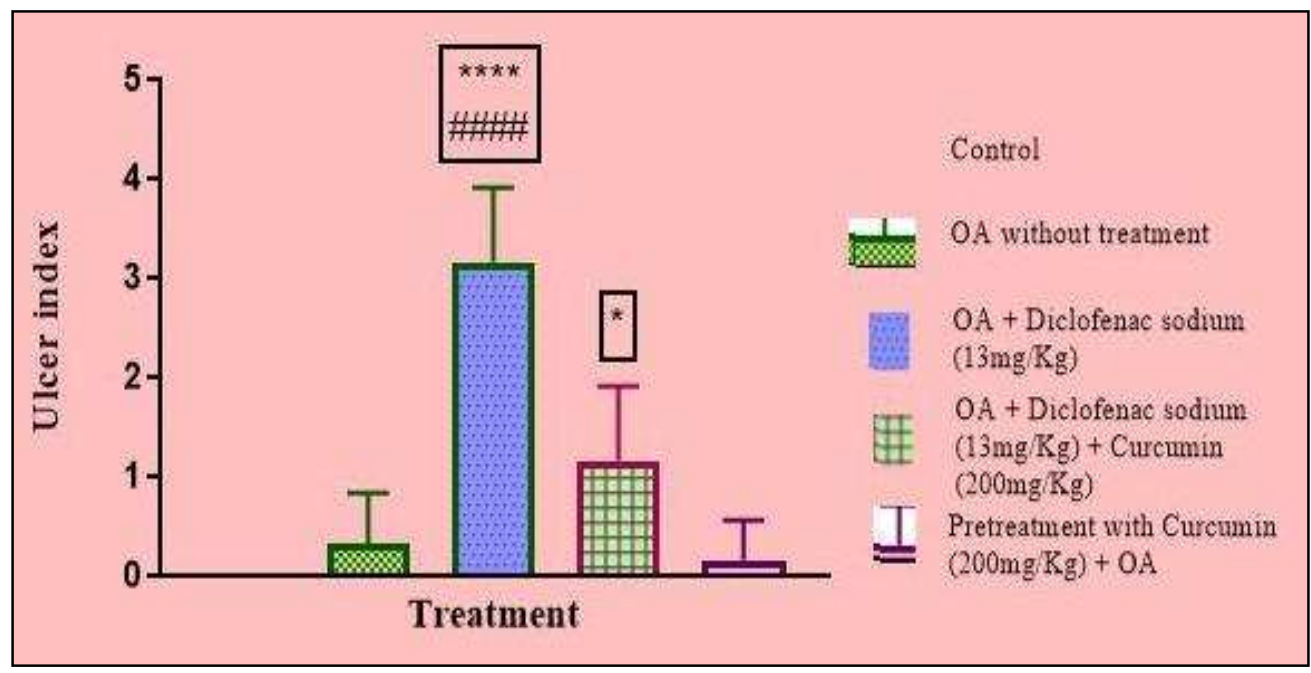

Fig. 1: Effect of various treatments on gastric ulcer formation in osteoarthritis $(\mathrm{OA})$ induced rats. All the data is expressed in mean \pm SD, $\mathrm{n}=6$. Control group animals have shown 0 zero ulcers. The ulcers were prominently increased in group III $\mathrm{OA}$ induced animals which were treated with diclofenac sodium $(p<0.001)$. There is a significant decrease in the ulcer formation in group IV ( $p<0.05) 0 A$ induced animals which were treated with diclofenac sodium and curcumin. Group $\mathrm{V}$ animals pre-treated with curcumin have shown fewer incidences of ulcers. $\mathbf{O A}=$ osteoarthritis 


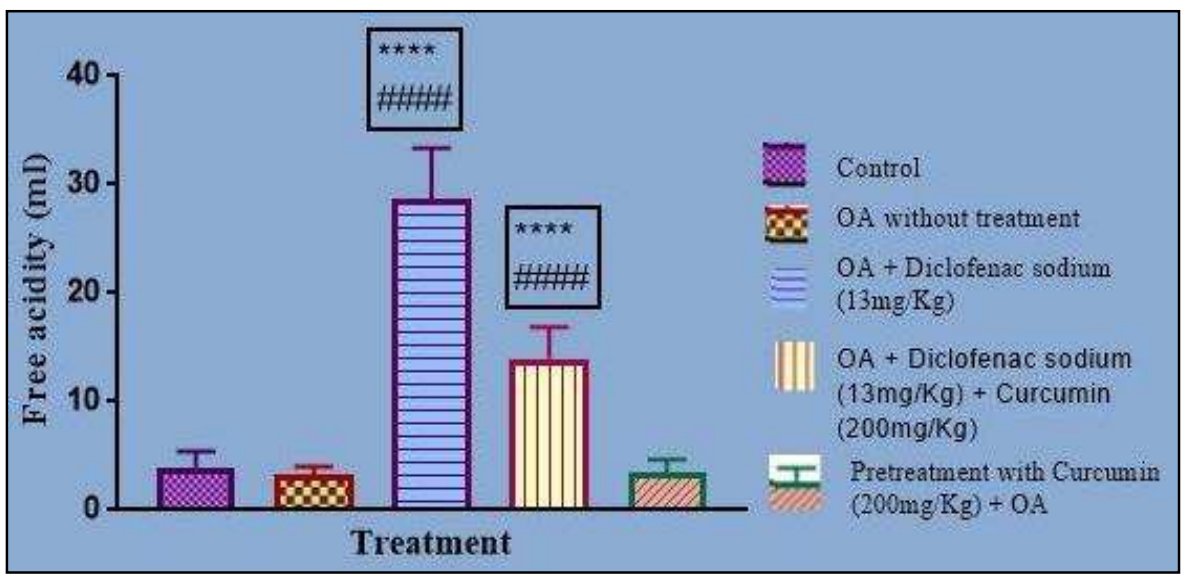

Fig. 2: Effect of various treatments on free acidity in osteoarthritis (OA) induced rats. All the data are expressed in mean $\pm S D, n=6$. The free acidity value has increased significantly in Group III $(p<0.001)$ animals which were treated with diclofenac sodium alone when compared to Group I (control animals) and II (OA induced animals without treatment) and it has decreased in group IV OA induced animals treated with diclofenac sodium and curcumin and group $\mathrm{V}$ animals pre-treated with curcumin when compared to group III. $\mathrm{OA}=\mathrm{osteoarthritis}$

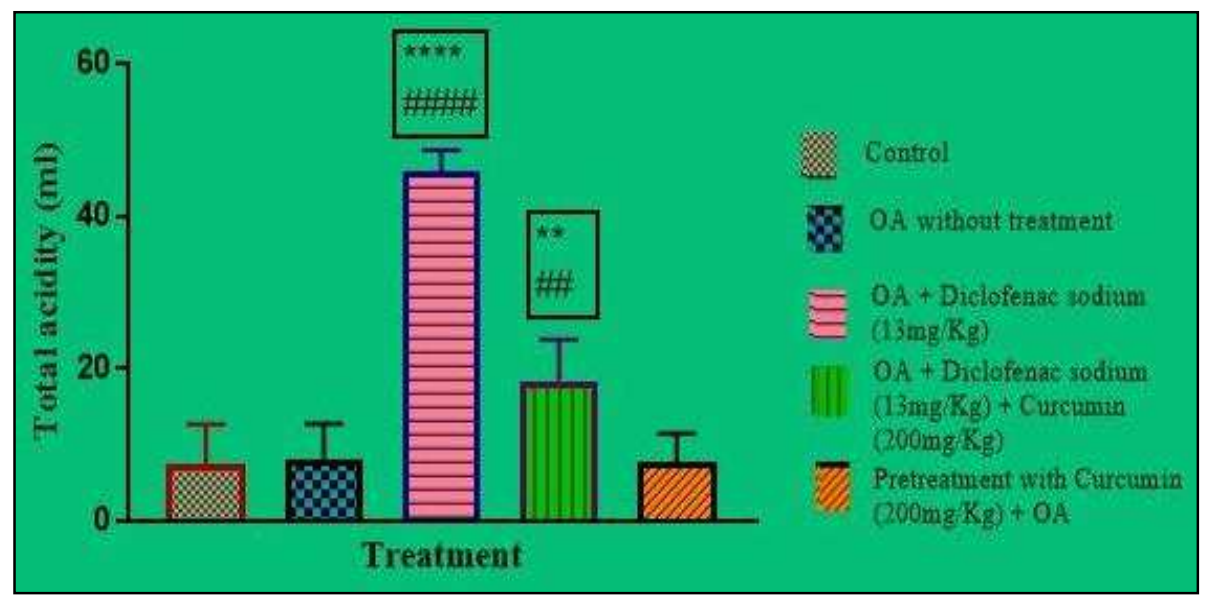

Fig. 3: Effect of various treatments on total acidity in osteoarthritis $(O A)$ induced rats. All the data are expressed in mean $\pm S D, n=6$. Total acidity value has increased significantly $(p<0.001)$ in group III $0 A$ induced animals treated with diclofenac sodium alone when compared to group I control animals and II OA induced animals without treatment whereas it is decreased in group IV $0 A$ induced animals treated with the combination of diclofenac sodium and curcumin and group $V$ animals which were pretreated with curcumin when compared to group III. $\mathrm{OA}=$ osteoarthritis

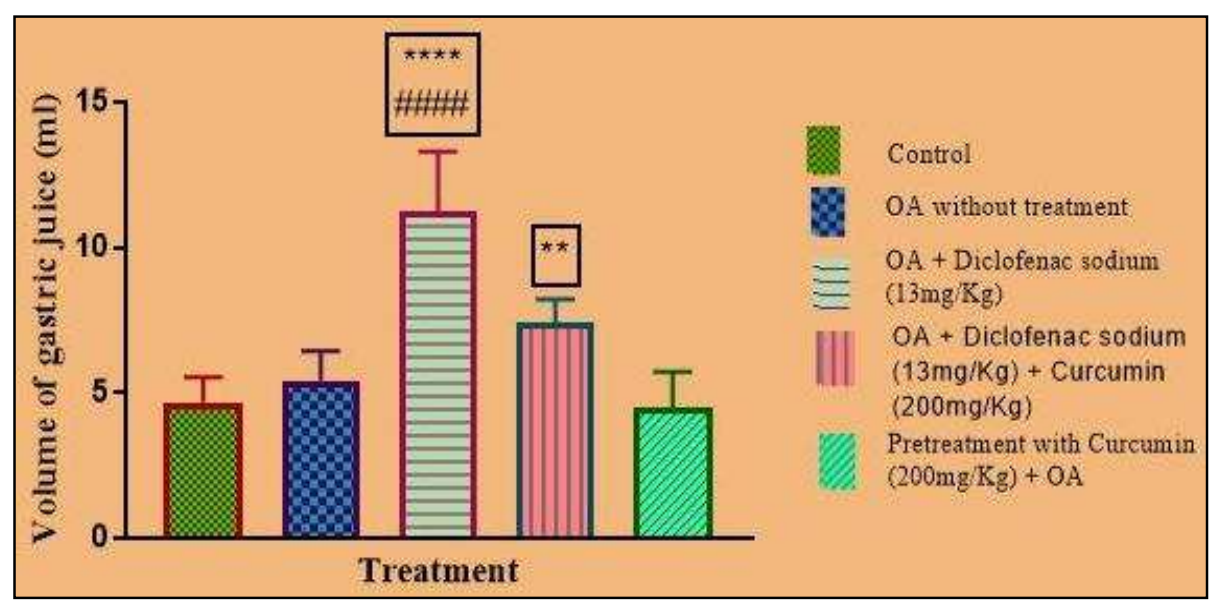

Fig. 4: Effect of various treatments on volume of gastric juice in osteoarthritis (OA) induced rats. All the data are expressed in mean \pm SD, $n=6$. The volume of gastric juice is increased significantly in group III $(p<0.001) 0 A$ induced animals which were treated and group IV $(p<0.01) 0 A$ induced animals treated with the combination of diclofenac sodium and curcumin whereas it decreased in group IV and group $\mathrm{V}$ animals which were pretreated with curcumin before inducing $\mathrm{OA}$ when compared to group III. $0 \mathrm{~A}=$ osteoarthritis 


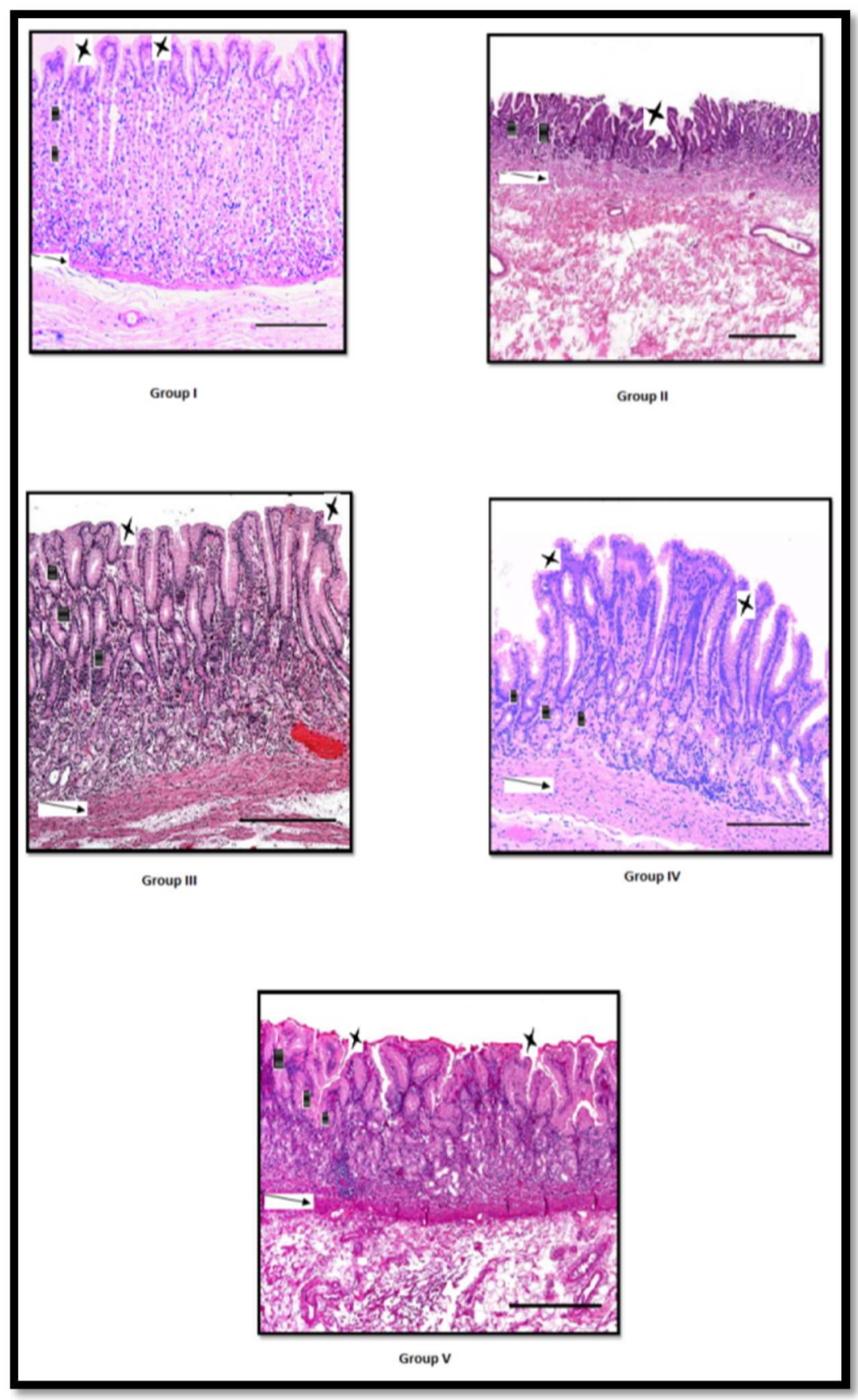

Fig. 5: Group I-From the above fig. it is evident that the stomach of animals belonging to control group show distinct mucosa layer (dots representing mucosa) with intact submucosa (arrow mark showing submucosa) and muscularis layer without any necrotic areas. The villa is preserved and no signs of ulceration (represented by star mark) were seen. Group II-osteoarthritis induced animals with no treatment have shown normal tissue of stomach with intact submucosa (represented by arrow mark) and muscularis without necrosis. There was no ulceration. The villi appear to be normal (represented by star mark). Group III-0steoarthritis induced animals treated with diclofenac sodium has shown marked changes in the tissue with severe ulceration exposing submucosa (arrow mark representing submucosa) layer with damaged villi (star representing villi). The muscularis layer has shown widespread atrophic gastritis with blood vessels showing intimal thickening (red mark). There was a severe mucosal degeneration with ulcerated area. Group IV-0steoarthritis induced animals treated with the combination of diclofenac sodium and curcumin has shown very few ulcers with well-preserved mucosa (dots representation), intact submucosa (arrow mark) and distinct muscularis beneath. Villi appear to be normal. Group V-Animals pretreated with curcumin and induced with Osteoarthritis has also shown the intact structure of stomach with less damage. The mucosa

(dots), submucosa (represented by the arrow) and muscularis were distinct. All the cells were found to be normal

\section{DISCUSSION}

Alleviating the pain is the main goal in the treatment of osteoarthritis. It is accomplished by using Non-steroidal antiinflammatory drugs like diclofenac sodium, naproxen, ibuprofen etc.
Gastrointestinal irritation and ulcers are the major limitations of the usage of these NSAIDs. NSAIDs cause gastric injury both by prostaglandin-dependent and prostaglandin-independent mechanisms. They cause damage to the stomach mainly by acting as an irritant, interfering with the defense and repair mechanism of 
mucosa and epithelium. Prostaglandins, Nitric oxide synthesis, and enteric nervous system are the key regulators which maintain the integrity of mucosa. Usage of NSAIDs compromises the mucosal blood flow, mucous secretion, and hydrophobic environment. They also impair restitution process interfering with the homeostasis. Three defense mechanisms are involved in gastric mucosa [6, 7].

The first defense mechanism consists of a barrier layer which is made up of mucosa, bicarbonates, and phospholipids which protects the gastric mucosa from noxious agents. The mucosa secreted by the epithelial layer and bicarbonate ions together maintain the neutral $\mathrm{pH}$ when there is an excess of gastric acid secretion making it acidic. The second mechanism comprises of epithelial cells. These cells prevent the diffusion of $\mathrm{H}^{+}$ions into them because of the tight junctions between them. The damaged epithelial cells will be shed forming a protective layer. The cells which are below get attached to mucous and migrate to damaged area helping in restoring the damaged cells-the process is called as restitution. The third mechanism involves high vascularity of mucosa which helps in supplying the required amount of oxygen and nutrients so that they can release an adequate amount of mucous and permeability. All these defense mechanisms are regulated and maintained by synthesis of prostaglandins. As NSAIDs are COX inhibitors, they reduce the pain by inhibiting the prostaglandin synthesis which is the key regulators of the defense mechanisms in the mucous membrane. Due to deficiency of prostaglandins during the usage of NSAIDs, the mucosal barrier and mucosal integrity are lost leading to the gastric injury.

Prostaglandin independent mechanism is explained by "Trapping theory" [8]. As NSAIDs are weak organic acids, they are unionized and lipophilic in the gastric environment. As these molecules get rapidly absorbed, they get into the gastric mucosal cell membrane where the $\mathrm{pH}$ is neutral. NSAIDs get the ionized and lipophobic form. Due to this nature, they get trapped and accumulate in the cell causing gastric injury. NSAIDs inhibit the mitochondrial metabolic pathways i. e oxidative phosphorylation to decreases the mitochondrial transmembrane potential (MTP) that results in releasing of cytochrome $C$ from mitochondria into the cytoplasm [9, 10]. Cytochrome $\mathrm{c}$ is the component which is involved in the initiation of apoptosis. It also results in the release of ROS, such as superoxides $\left(\mathrm{O}_{2}^{-}\right)$and hydrogen peroxide $\left(\mathrm{H}_{2} \mathrm{O}_{2}\right)$ which causes activation of caspase 9 and caspase 3 , cellular lipid peroxidation finally resulting in programmed cell death process called as apoptosis. The uncoupling of mitochondria also results in decreased ATP concentration which causes leakage of calcium ions leading to a loss of cellular osmotic balance and control over intracellular functions resulting in increased permeability and subsequent neural damage in the intestine. As there is disturbance or alteration in the permeability of mucosal layer in the stomach as well as in intestine, the cells are uncovered and are exposed to all the contents of the lumen-like bile acids, proteolytic enzymes, pancreatic enzymes, intestinal bacteria causing neutrophil chemotaxis. Neutrophil activation results in inflammation and ulceration. Due to these effects caused by NSAIDs, there is an immediate need for an alternative therapy or an adjuvant therapy which can combat this effect and help in effectively treating the pain in Osteoarthritis or any chronic inflammatory disease. Herbal medicine is considered to be safest alternative or adjuvant because of their easy availability, high safety with no adverse effects. In this study due to its antiinflammatory effect, curcumin is taken as an adjuvant therapy.

Curcumin has considerable and significant gastroprotective and antiulcerogenic effects. Its antiulcer activity is due to its inhibition of hypersecretion of gastric acid, total peroxides, IL-6, apoptotic enhancers and also on pepsin secretion. The phenolic $(\mathrm{OH})$ group in the structure of curcumin plays an important role in anticancer activity which in turn is because of its antioxidant activity. NSAIDS inactivates gastric peroxidase enzyme and causes ROS mediated gastric mucosal injury. Curcumin protects peroxide enzymes from inactivation, removes $\mathrm{H}_{2} \mathrm{O}_{2}$ from the cells and decreases gastric damage [11]. The gastric cellular injury is also enhanced by inflammation with the release of several inflammatory cytokines and leukocyte adhesions [12]. Curcumin, as it has anti-inflammatory activity, inhibits inflammation and gastric ulceration. Curcumin in addition specifically acts on COX-2 and Thromboxane $\mathrm{A}_{2}\left(\mathrm{TXA}_{2}\right)$ when compared to COX-1 without affecting the synthesis of protective prostaglandins; it also inhibits LOX (lipoxygenase), induced nitric oxide synthase (iNOS) enzymes which are also important mediators of inflammation. All these activities of curcumin serve as an evidence for its anti-ulcer and gastroprotective action. Curcumin induces Heme-oxygenase-1(H0-1) enzyme expression through the Nrf2/ARE pathway which is a protective mechanism against oxidative stress condition which exists in osteoarthritis [13]. It also induces the expression of $\mathrm{HO}-1$ by decreasing the level of Bach-1which is the repressor factor of $\mathrm{H0}-1$. Deficiency of Bach-1 increases the expression of HO-1 thereby inducing antioxidant action [14]. Curcumin also inhibits TNF- $\alpha$ induced ICAM-1 expression which is responsible for leucocytes infiltration promoting inflammation [15]. In addition, curcumin also stimulates and activates transcription factors responsible for the expression of glutathione, catalase, superoxide dismutase which are important antioxidant systems $[16,17]$. Therefore, curcumin can reduce leukocyte infiltration and can modulate different pathways of oxidative stress thereby acting as a potent antioxidant. It also decreases expression of thioredoxin-interacting protein (TXNIP) which is actually an oxidative stress mediator. It is a potent regulator of redox systems [18]. In hypoxia conditions, TXNIP is upregulated attributing to oxidative stress. Histopathological studies also support the activity of curcumin as a gastroprotective agent.

\section{CONCLUSION}

Curcumin was found to have gastroprotective and anti ulcerogenic activity in this study and therefore it can serve as an adjuvant therapy to NSAIDs to alleviate the pain. In addition due to its antiinflammatory properties, it can effectively help in treating Osteoarthritis thereby improving the quality of life in Osteoarthritis patients. As it is an herbal drug, it is safe even in age-old patients in whom Osteoarthritis is the major disorder in today's world.

\section{ACKNOWLEDGEMENT}

The corresponding author would like to thank Dr. B. Bindu Madhavi, Dr. P. Ajay Godwin, Mr. Shaik Imran and Management of St. Pauls College of Pharmacy for their help, support and encouragement.

\section{AUTHORS CONTRIBUTIONS}

Nasreen Sulthana-Conducted the experiment and prepared the manuscript.

Dr. K. Vijaya-Helped in designing and conducting the experiment.

\section{CONFLICT OF INTERESTS}

\section{Declared none}

\section{REFERENCES}

1. Aigner T. Osteoarthritis. Curr Opin Rheumatol 2007;19:427-8.

2. Kellgren JH, Moore R. Generalized osteoarthritis and Heberden's nodes. Br Med J 1952;1:181.

3. Uivarosi V. Metal complexes of quinolone antibiotics and their applications: an update. Molecules 2013;18:11153-97.

4. Shay H, Kamarow SA, Fels SS, Mersanze D, Guenstein M, Siplet HA. A simple method for the uniform production of gastric ulceration in the rat. Gastroenterol 1945;5:43-61.

5. Sener G, Paskaloglu K, Ayanoglu-dülger G. Protective effect of increasing doses of famotidine, omeprazole, lansoprazole, and melatonin against ethanol-induced gastric damage in rats. Indian J Pharmacol 2004;36:171-4.

6. Turnberg LA. Gastric mucosal defence mechanisms: a brief review. Scandinavian J Gastroenter 1985;20:37-40.

7. Hoy B, Brandstetter H, Wessler S. The stability and activity of recombinant Helicobacter pylori HtrA under stress conditions. J Basic Microbiol 2013;53:402-9.

8. Castro GA, Carvalho JE, Tinti SV, Possenti A, Sgarbieri VC. Antiulcerogenic effect of a whey protein isolate and collagen hydrolysates against ethanol ulcerative lesions on oral administration to rats. J Med Food 2010;13:83-90.

9. Matsui H, Shimokawa O, Kaneko T, Nagano Y, Rai K, Hyodo I. The pathophysiology of nonsteroidal antiinflammatory drug (NSAID) induced mucosal injuries in stomach and small intestine. J Clin Biochem Nutr 2011;48:107-11. 
10. Bjarnason I, Hayllar J. Early pathogenic events in NSAIDinduced gastrointestinal damage. Ital J Gastroenterol 1996;28 Suppl 4:19-22.

11. Higuchi $\mathrm{K}$, Umegaki E, Watanabe T. Present status and strategy of NSAIDs-induced small bowel injury. J Gastroenterol 2009; 44:879-88.

12. Thong-Ngam D, Choochuai S, Patumraj S, Chayanupatkul M, Klaikeaw N. Curcumin prevents indomethacin-induced gastropathy in rats. World J Gastroenterol 2012;18:1479-84.

13. Yadav SK, Sah AK, Jha RK, Sah P, Shah DK. Turmeric (curcumin) remedies gastroprotective action. Pharmacogn Rev 2013;7:42-6.

14. Balogun E, Hoque M, Gong P. Curcumin activates the haem oxygenase-1 gene via regulation of $\mathrm{Nrf} 2$ and the antioxidantresponsive element. Biochem J 2003;371:887-95.
15. Blandine Poulet, Frank Beier. Targeting oxidative stress to reduce osteoarthritis. Arthritis Res Ther 2016;18:32.

16. Youn GS, Kwon DJ, Ju SM, Choi SY, Park J. Curcumin ameliorates TNF- $\alpha$-induced ICAM-1 expression and subsequent THP-1 adhesiveness via the induction of heme oxygenase- 1 in the HaCaT cells. BMB Rep 2013;46:410-5.

17. Rogers NM, Stephenson MD, Kitching AR, Horowitz JD, Coates PT. Amelioration of renal ischaemia-reperfusion injury by liposomal delivery of curcumin to renal tubular epithelial and antigen-presenting cells. Br J Pharmacol 2012;166:194-209.

18. Trujillo J, Chirino YI, Molina-Jijon E, Anderica-Romero AC, Tapia E, Pedraza-Chaverri J. Renoprotective effect of the antioxidant curcumin: recent findings. Redox Biol 2013;1:448-56. 